\title{
6 \\ Long-range interacting systems in the unconstrained ensemble
}

\author{
Ivan Latella, ${ }^{1, *}$ Agustín Pérez-Madrid, ${ }^{2, \dagger}$ Alessandro Campa, ${ }^{3, \dagger}$ Lapo Casetti, ${ }^{4,5,8}$ and Stefano Ruffo ${ }^{6, \|}$ \\ ${ }^{1}$ Laboratoire Charles Fabry, UMR 8501, Institut d'Optique, CNRS, Université Paris-Saclay, 2 Avenue Augustin Fresnel, \\ 91127 Palaiseau Cedex, France \\ ${ }^{2}$ Departament de Física de la Matèria Condensada, Facultat de Física, Universitat de Barcelona, Martí i Franquès 1, 08028 Barcelona, Spain \\ ${ }^{3}$ Complex Systems and Theoretical Physics Unit, Health and Technology Department, Istituto Superiore di Sanità, \\ and INFN Roma 1, Viale Regina Elena 299, 00161 Roma, Italy \\ ${ }^{4}$ Dipartimento di Fisica e Astronomia and CSDC, Università di Firenze, and INFN, Sezione di Firenze, via G. Sansone 1, \\ 50019 Sesto Fiorentino (FI), Italy \\ ${ }^{5}$ INAF-Osservatorio Astrofisico di Arcetri, Largo E. Fermi 5, 50125 Firenze, Italy \\ ${ }^{6}$ SISSA, via Bonomea 265, ISC-CNR and INFN, 34136 Trieste, Italy \\ (Received 17 November 2016; published 23 January 2017)
}

\begin{abstract}
Completely open systems can exchange heat, work, and matter with the environment. While energy, volume, and number of particles fluctuate under completely open conditions, the equilibrium states of the system, if they exist, can be specified using the temperature, pressure, and chemical potential as control parameters. The unconstrained ensemble is the statistical ensemble describing completely open systems and the replica energy is the appropriate free energy for these control parameters from which the thermodynamics must be derived. It turns out that macroscopic systems with short-range interactions cannot attain equilibrium configurations in the unconstrained ensemble, since temperature, pressure, and chemical potential cannot be taken as a set of independent variables in this case. In contrast, we show that systems with long-range interactions can reach states of thermodynamic equilibrium in the unconstrained ensemble. To illustrate this fact, we consider a modification of the Thirring model and compare the unconstrained ensemble with the canonical and grand-canonical ones: The more the ensemble is constrained by fixing the volume or number of particles, the larger the space of parameters defining the equilibrium configurations.
\end{abstract}

DOI: 10.1103/PhysRevE.95.012140

\section{INTRODUCTION}

Systems with long-range interactions may display a notable thermodynamic behavior that distinguishes them from those where the interactions are short ranged [1-3]. Long-range interactions are those for which the range is comparable with the size of the system, regardless of how large the system is, as it occurs, for instance, in self-gravitating systems [4-11], plasmas [12,13], fluid dynamics [14,15], and some spin systems [16]. Because of these interactions, the system may remain trapped in nonequilibrium quasistationary states [17] whose lifetimes depend on the number of particles and diverge in the limit $N \rightarrow \infty$. For large but finite numbers of particles in a very long time limit, however, the system eventually evolves towards states of thermodynamic equilibrium [18] that can be described within the usual framework of ensemble theory. At equilibrium, such systems may present ensemble inequivalence [6,19-21] associated to anomalies in the concavity of the thermodynamic potentials [21,22]. In particular, a negative heat capacity in the microcanonical ensemble [5,6,23] is due to an anomaly in the concavity of the entropy as a function of the energy. In addition, systems with long-range interactions, in general, do not obey the usual Gibbs-Duhem equation [24,25] (see Ref. [26] for a discussion regarding

\footnotetext{
*ivan.latella@institutoptique.fr

†agustiperezmadrid@ub.edu

¥alessandro.campa@iss.infn.it

§lapo.casetti@unifi.it

"ruffo@sissa.it
}

self-gravitating systems). This fact is deeply related with the nonadditive character that long-range interactions confer to these systems [25]. Actually, nonadditivity is responsible for the remarkable behavior observed in long-range interacting systems [1], as well as for the physics behind the subject we discuss in this paper: the appearance of a new equilibrium statistical ensemble in the thermodynamic limit.

In this paper we focus on the thermodynamics of long-range interacting systems under completely open conditions. A completely open system can exchange heat, work, and matter with its surroundings. That is, the energy, volume, and number of particles of the system fluctuate under completely open conditions. Thus, the control parameters that specify the thermodynamic state of the system are temperature, pressure, and chemical potential. These control parameters are properties of a suitable reservoir that weakly interacts with the system, in the sense that, by means of some mechanism, it supplies heat, work, and matter to the system but can be assumed not to be coupled by long-range interactions. It is worth mentioning that under these conditions the system is not enclosed by rigid walls. Instead, the system could be confined by an external field exerting pressure on it; e.g., tidal forces produced by surrounding objects in self-gravitating systems or magnetic fields acting on plasmas [27]. Furthermore, the unconstrained ensemble is the statistical ensemble that describes a completely open system (in the literature, this ensemble is also termed generalized [28]). It was introduced by Guggenheim [29], but it did not receive much attention due to its lack of application in standard macroscopic systems. If the interactions in the system are short ranged, the free energy associated to this ensemble is vanishingly small when the number of particles is large [29]. 
This is a consequence of the fact that temperature, pressure, and chemical potential cannot be treated as independent variables because they are related by the Gibbs-Duhem equation. Thus they are not, taken together, suitable control parameters for macroscopic systems with short-range interactions.

In case that the limit of large number of particles is not assumed, however, the situation changes, as pointed out by Hill [30]. Small systems may have an extra degree of freedom which permits that equilibrium configurations in completely open conditions can be realized [30-33]. Nevertheless, when the size of the system is increased, the usual behavior of macroscopic systems is obtained [30,34,35], as long as the interactions remain short ranged.

Our aim here is to show that equilibrium configurations with a large number of particles may be realized in the unconstrained ensemble if the interactions in the system are long ranged. Moreover, we argue that the replica energy [25] is the appropriate free energy defining such configurations in this ensemble. Thus, in Sec. II, we will first describe this ensemble and make the appropriate connection with the thermodynamics. In Sec. III we introduce a solvable model, whose thermodynamics in the unconstrained ensemble is discussed in Sec. III A. In Secs. III B and III C, this model is studied in the grand-canonical and canonical ensembles, respectively. In addition, some thermodynamic relations associated with the replica energy are analyzed in Sec. IV, and our conclusions are presented in Sec. V.

\section{THE UNCONSTRAINED ENSEMBLE}

To make our point clear, it is worth it to briefly review the thermostatistics of completely open systems by establishing its connection to the unconstrained ensemble. The description of the unconstrained ensemble presented in this section is based on Refs. [28] and [30], which the reader is referred to for an extended discussion (see also Refs. [29,32,36,37]).

The energy, volume, and number of particles of a completely open system are quantities that fluctuate due the interaction of the system with its surroundings. Let us consider the probability $p_{i}(V, N)$ of the configuration of a system that is found in the state $i$ with energy $E_{i}$ and possesses $N$ particles in a volume $V$. This probability is an exponential of the form [28]

$$
p_{i}(V, N)=\frac{\exp \left[-\alpha N-\beta E_{i}(V, N)-\gamma V\right]}{\Upsilon},
$$

where $\alpha, \beta$, and $\gamma$ are parameters that will be identified below and $\Upsilon$ is the associated partition function given by

$$
\Upsilon=\sum_{i, V, N} \exp \left[-\alpha N-\beta E_{i}(V, N)-\gamma V\right] .
$$

Here discrete variables are used for simplicity. The ensemble average $\bar{E}$ of the internal energy $E$ is thus obtained as $\bar{E}=\sum_{i, V, N} E_{i}(V, N) p_{i}(V, N)$, while the average number of particles $\bar{N}$ and the average volume $\bar{V}$ $\operatorname{read} \bar{N}=\sum_{i, V, N} N p_{i}(V, N)$ and $\bar{V}=\sum_{i, V, N} V p_{i}(V, N)$, respectively. Now let us consider an infinitesimal change of the average internal energy in terms of changes in the probability,

$$
d \bar{E}=\sum_{i, V, N} E_{i}(V, N) d p_{i}(V, N),
$$

where the coefficients $E_{i}(V, N)$ are assumed constant. Hence, using Eq. (1) to express $E_{i}(V, N)$ and the condition $\sum_{i, V, N} d p_{i}(V, N)=0$, Eq. (3) can be rewritten as

$$
d \bar{E}=-\frac{1}{\beta} d\left[\sum_{i, V, N} p_{i}(V, N) \ln p_{i}(V, N)\right]-\frac{\alpha}{\beta} d \bar{N}-\frac{\gamma}{\beta} d \bar{V} .
$$

If Eq. (4) is compared with the thermodynamic equation

$$
d \bar{E}=T d S-P d \bar{V}+\mu d \bar{N},
$$

where $T$ is the temperature, $P$ is the pressure, and $\mu$ is the chemical potential, one then recognizes $k_{B} T=1 / \beta$, $\mu=-\alpha / \beta, P=\gamma / \beta$, and the entropy

$$
S=-k_{B} \sum_{i, V, N} p_{i}(V, N) \ln p_{i}(V, N),
$$

where $k_{B}$ is the Boltzmann constant. Therefore, the probability (1) leads to the correct thermostatistical description of completely open systems. Furthermore, substituting Eq. (1) in Eq. (6) with the previous identifications, one gets

$$
\mathscr{E}=\bar{E}-T S+P \bar{V}-\mu \bar{N},
$$

where we have introduced the free energy

$$
\mathscr{E}(T, P, \mu)=-k_{B} T \ln \Upsilon(T, P, \mu),
$$

called replica energy [25] or subdivision potential [30]. By differentiation of Eq. (7) and using Eq. (5), one obtains

$$
d \mathscr{E}=-S d T+\bar{V} d P-\bar{N} d \mu .
$$

Notice that, as a consequence of Eq. (9), one has

$$
\begin{aligned}
& \left(\frac{\partial \mathscr{E}}{\partial T}\right)_{P, \mu}=-S, \\
& \left(\frac{\partial \mathscr{E}}{\partial P}\right)_{T, \mu}=\bar{V}, \\
& \left(\frac{\partial \mathscr{E}}{\partial \mu}\right)_{T, P}=-\bar{N} .
\end{aligned}
$$

Moreover, the unconstrained partition function can be written as

$$
\Upsilon(T, P, \mu)=\sum_{V, N} Z(T, V, N) e^{\mu N /\left(k_{B} T\right)} e^{-P V /\left(k_{B} T\right)},
$$

where

$$
Z(T, V, N)=\sum_{i} e^{-E_{i}(V, N) /\left(k_{B} T\right)}
$$

is the canonical partition function. From Eq. (13), it is straightforward to connect $\Upsilon$ with the partition function of another ensemble, e.g., one has

$$
\Upsilon(T, P, \mu)=\sum_{V} \Xi(T, V, \mu) e^{-P V /\left(k_{B} T\right)},
$$

where $\Xi(T, V, \mu)$ is the grand-canonical partition function.

For macroscopic systems with short-range interactions in the thermodynamic limit, the internal energy and the other thermodynamic potentials are linear homogeneous functions of the extensive variables and thus, from Eq. (7), one obtains $\mathscr{E}=0$, since in this case the Gibbs free energy $G=\bar{E}-T S+P \bar{V}$ 
is equal to $\mu \bar{N}$. We note that in this case it does not mean that $\Upsilon=1$ but that $\mathscr{E}$ is negligible in the thermodynamic limit [28]. Thus, for this kind of system, Eq. (9) reduces to the well-known Gibbs-Duhem equation. The interesting fact is that Eq. (7) indicates the route to deal with the more general situation in which the internal energy is not a linear homogeneous function of $S, V$, and $N$, as it happens with systems with long-range interactions. This is the case we are concerned with, for which the replica energy, in general, differs from zero.

The above considerations are directly related to the fact that for an ensemble of $\mathscr{N}$ replicas of the system with total energy $E_{t}=\mathscr{N} E$, entropy $S_{t}=\mathscr{N} S$, volume $V_{t}=\mathscr{N} V$, and number of particles $N_{t}=\mathscr{N} N, \mathscr{E}$ can be interpreted as the energy gained by the ensemble when the number of replicas changes holding $S_{t}, V_{t}$ and $N_{t}$ constant. Formally [30],

$$
\mathscr{E}=\left(\frac{\partial E_{t}}{\partial \mathscr{N}}\right)_{S_{t}, V_{t}, N_{t}} .
$$

When the single-system energy $E$ is a linear homogeneous function of $S, V$, and $N$ (additive systems), one has $E_{t}=\mathscr{N} E\left(S_{t} / \mathscr{N}, V_{t} / \mathscr{N}, N_{t} / \mathscr{N}\right)=E\left(S_{t}, V_{t}, N_{t}\right)$ and, therefore, Eq. (16) leads to $\mathscr{E}=0$. If $E$ is not a linear homogeneous function of $S, V$, and $N$ (nonadditive systems), then Eq. (16) requires $\mathscr{E} \neq 0[25]$.

Finally, we want to stress that Eqs. (7) and (9)-(12) are relations at a thermodynamic level. That is, for any other ensemble, corresponding to the actual physical conditions specified by the given control parameters, an analogous set of equations can be obtained from the appropriate free energy [30]. The replica energy will emerge also in those cases, although it will not be the free energy associated to the partition function for that ensemble. However, since in any case $\mathscr{E}$ vanishes when the system is additive, it can always be seen as a measure of the nonadditivity of the system [25].

\section{THE MODIFIED THIRRING MODEL}

We introduce here a model that, as discussed in the following, can attain equilibrium configurations under completely open conditions. Consider a system of $N$ particles of mass $m$ enclosed in a volume $V$ with a Hamiltonian of the form

$$
\mathcal{H}(\boldsymbol{p}, \boldsymbol{q})=\sum_{i=1}^{N} \frac{\left|\boldsymbol{p}_{i}\right|^{2}}{2 m}+\sum_{i>j}^{N} \phi\left(\boldsymbol{q}_{i}, \boldsymbol{q}_{j}\right)
$$

where $\boldsymbol{p}_{i} \in \mathbb{R}^{3}$ and $\boldsymbol{q}_{i} \in \mathbb{R}^{3}$ are the momentum and position of particle $i$, respectively, $\phi\left(\boldsymbol{q}_{i}, \boldsymbol{q}_{j}\right)$ is the interaction potential, $\boldsymbol{p}=\left(\boldsymbol{p}_{1}, \ldots, \boldsymbol{p}_{N}\right)$, and $\boldsymbol{q}=\left(\boldsymbol{q}_{1}, \ldots, \boldsymbol{q}_{N}\right)$. The interactions in this model are defined by

$$
\phi\left(\boldsymbol{q}_{i}, \boldsymbol{q}_{j}\right)=-2 v\left[\theta_{V_{0}}\left(\boldsymbol{q}_{i}\right) \theta_{V_{0}}\left(\boldsymbol{q}_{j}\right)+b \theta_{V_{1}}\left(\boldsymbol{q}_{i}\right) \theta_{V_{1}}\left(\boldsymbol{q}_{j}\right)\right],
$$

where $v>0$ and $b$ are constants, and we have introduced the functions

$$
\begin{aligned}
& \theta_{V_{0}}\left(\boldsymbol{q}_{i}\right)=\left\{\begin{array}{ll}
1 & \text { if } \boldsymbol{q}_{i} \in V_{0} \\
0 & \text { if } \boldsymbol{q}_{i} \notin V_{0}
\end{array},\right. \\
& \theta_{V_{1}}\left(\boldsymbol{q}_{i}\right)=\left\{\begin{array}{ll}
1 & \text { if } \boldsymbol{q}_{i} \notin V_{0} \\
0 & \text { if } \boldsymbol{q}_{i} \in V_{0}
\end{array} .\right.
\end{aligned}
$$

Here $V_{0}$ is the volume of an internal region of the system such that $V_{0}<V$, and $V_{1}$ is the volume of the region outside $V_{0}$ so $V_{1}=V-V_{0}$. In this way, $V_{0}$ is a parameter of the interaction potential that does not depend on the state of the system. Hence, the total potential energy in the large $N$ limit is given by

$$
\hat{W}\left(N_{0}, N_{1}\right) \equiv \sum_{i>j}^{N} \phi\left(\boldsymbol{q}_{i}, \boldsymbol{q}_{j}\right)=-v\left(N_{0}^{2}+b N_{1}^{2}\right),
$$

where $N_{0}$ is the number of particles in $V_{0}$ and $N_{1}=N-N_{0}$ is the number of particles in $V_{1}$ for a given configuration. Notice that $N_{0}=N_{0}(\boldsymbol{q})$ and $N_{1}=N_{1}(\boldsymbol{q})$, so $\hat{W}$ depends implicitly on the position of all particles. This model is a modification of the Thirring model [6], which is obtained in the particular case $b=$ 0 . Furthermore, the model is solvable in the different statistical ensembles, and below we focus on the unconstrained ensemble and compare it with the canonical and grand-canonical cases. In the following, it will become clear why we consider this modification of the model.

\section{A. The unconstrained ensemble}

Here we study the modified Thirring model in the unconstrained ensemble. Thus, we will assume that the system is in contact with a reservoir characterized by fixed temperature $T$, pressure $P$, and chemical potential $\mu$.

Consider the canonical partition function for this model, which is given by

$$
\begin{aligned}
Z(T, V, N) & =\int \frac{d^{3 N} \boldsymbol{q} d^{3 N} \boldsymbol{p}}{h^{3 N} N !} e^{-\beta \mathcal{H}(\boldsymbol{p}, \boldsymbol{q})} \\
& =\int \frac{d^{3 N} \boldsymbol{q}}{N !} \frac{e^{-\beta \hat{W}\left(N_{0}, N_{1}\right)}}{\lambda_{T}^{3 N}},
\end{aligned}
$$

where $\lambda_{T}=h / \sqrt{2 \pi m T}$ is the thermal wavelength, $h$ being a constant, and hereafter we take units where $k_{B}=1$. Thus, using Eq. (13), the unconstrained partition function becomes (we take $V$ as a continuous variable)

$$
\begin{aligned}
\Upsilon(T, P, \mu)= & \int d V \sum_{N} \int \frac{d^{3 N} \boldsymbol{q}}{N !} \\
& \times \lambda_{T}^{-3 N} e^{-\beta \hat{W}\left(N_{0}, N_{1}\right)} e^{\beta \mu N} e^{-\beta P V} .
\end{aligned}
$$

Following Thirring's method [6], the partition function can be computed by replacing the integral over coordinates with a sum over the occupation numbers in each region of the system,

$$
\int \frac{d^{3 N} \boldsymbol{q}}{N !} \rightarrow \sum_{N_{0}, N_{1}} \delta_{N, N_{0}+N_{1}} \frac{V_{0}^{N_{0}}}{N_{0} !} \frac{V_{1}^{N_{1}}}{N_{1} !}
$$

where the sum runs over all possible values of $N_{0}$ and $N_{1}$ and the Kronecker $\delta$ enforces the condition $N=N_{0}+N_{1}$. This leads to

$$
\Upsilon(T, P, \mu)=\int d V \sum_{N_{0}, N_{1}} e^{-\beta \hat{\mathscr{E}}\left(T, P, \mu, V, N_{0}, N_{1}\right)},
$$


where we have introduced (from now on we omit writing down explicitly the dependence on the control parameters)

$$
\begin{aligned}
\hat{\mathscr{E}}\left(V, N_{0}, N_{1}\right)= & \hat{W}\left(N_{0}, N_{1}\right)+P V-T \sum_{k} N_{k} \\
& +T \sum_{k} N_{k}\left[\ln \left(N_{k} \frac{\lambda_{T}^{3}}{V_{k}}\right)-\frac{\mu}{T}\right],
\end{aligned}
$$

with $k=0,1$, and we have used Stirling's approximation. Since the replica energy is given by $\mathscr{E}=-T \ln \Upsilon$, using the saddle-point approximation, one obtains

$$
\mathscr{E}=\inf _{\left\{V, N_{0}, N_{1}\right\}} \hat{\mathscr{E}}\left(V, N_{0}, N_{1}\right)
$$

Thus, minimization with respect to $V, N_{0}$, and $N_{1}$ requires that

$$
\begin{gathered}
P=\frac{T \bar{N}_{1}}{\bar{V}-V_{0}}, \\
\mu=-2 v \bar{N}_{0}+T \ln \left(\frac{\bar{N}_{0}}{V_{0}} \lambda_{T}^{3}\right), \\
\mu=-2 b \nu \bar{N}_{1}+T \ln \left(\frac{\bar{N}_{1}}{\bar{V}-V_{0}} \lambda_{T}^{3}\right),
\end{gathered}
$$

where $\bar{V}, \bar{N}_{0}$, and $\bar{N}_{1}$ are the values of the volume and the number of particles in each region that minimizes the replica energy. It is clear that $\bar{V}, \bar{N}_{0}$, and $\bar{N}_{1}$ are functions of $T, P$, and $\mu$, whose dependence is implicit through Eqs. (27)-(29). The mean value of the total number of particles is then given by $\bar{N}=\sum_{k} \bar{N}_{k}$. Moreover, the total potential energy for equilibrium configurations in the unconstrained ensemble becomes

$$
W \equiv \hat{W}\left(\bar{N}_{0}, \bar{N}_{1}\right)=-v\left(\bar{N}_{0}^{2}+b \bar{N}_{1}^{2}\right)
$$

Hence, notice that from Eqs. (28) and (29) one obtains

$$
\mu \bar{N}=T \sum_{k} \bar{N}_{k} \ln \left(\frac{\bar{N}_{k}}{V_{k}} \lambda_{T}^{3}\right)+2 W
$$

with $V_{1}=\bar{V}-V_{0}$. Therefore, using Eqs. (25) and (26), the replica energy takes the form

$$
\mathscr{E}=-W+P^{(\mathrm{e})} \bar{V},
$$

as given in Ref. [25], where $P^{(\mathrm{e})}=P-\bar{N} T / \bar{V}$ is the excess pressure.

We introduce the dimensionless variables

$$
v=\frac{V-V_{0}}{V_{0}}, \quad x_{0}=\frac{v N_{0}}{T}, \quad x_{1}=\frac{v N_{1}}{T},
$$

which will be denoted as $\bar{v}, \bar{x}_{0}$, and $\bar{x}_{1}$ when evaluated at $\bar{V}$, $\bar{N}_{0}$, and $\bar{N}_{1}$, respectively. In addition, we define the reduced pressure $p$ and the relative fugacity $z$ by

$$
p \equiv \frac{\nu V_{0}}{T^{2}} P, \quad z \equiv e^{\left(\mu-\mu_{0}\right) / T},
$$

where

$$
\mu_{0}=T \ln \left(\frac{T \lambda_{T}^{3}}{\nu V_{0}}\right)
$$

Since controlling $T, P$, and $\mu$ is equivalent to controlling $T$, $p$, and $z$, the latter set of variables can be taken as the set of control parameters in this ensemble. Using these variables, from Eqs. (27)-(29) one obtains

$$
\begin{gathered}
\bar{x}_{0}=z e^{2 \bar{x}_{0}}, \\
\bar{x}_{1}(p, z)=\frac{1}{2 b} \ln \left(\frac{p}{z}\right), \\
\bar{v}(p, z)=\frac{1}{2 b p} \ln \left(\frac{p}{z}\right),
\end{gathered}
$$

where Eq. (36) defines implicitly $\bar{x}_{0}=\bar{x}_{0}(z)$. Furthermore, let us consider the reduced replica energy $\varphi_{U}=v \mathscr{E} / T^{2}$ and the function $\hat{\varphi}_{U}=v \hat{\mathscr{E}} / T^{2}$, where, using Eqs. (33) and (34), the latter can be written as

$$
\begin{aligned}
\hat{\varphi}_{U}\left(v, x_{0}, x_{1}\right)= & x_{0}\left[\ln \left(\frac{x_{0}}{z}\right)-1\right]-x_{0}^{2}+p(v+1) \\
& +x_{1}\left[\ln \left(\frac{x_{1}}{v z}\right)-1\right]-b x_{1}^{2} .
\end{aligned}
$$

We note that with the dimensionless quantities, i.e., in the reduced replica energy and in Eqs. (36)-(38), the temperature does not appear; therefore $T$ acts as a simple scaling factor. The condition (26) becomes

$$
\varphi_{U}\left(\bar{v}, \bar{x}_{0}, \bar{x}_{1}\right)=\inf _{\left\{v, x_{0}, x_{1}\right\}} \hat{\varphi}_{U}\left(v, x_{0}, x_{1}\right) .
$$

Since the Hessian matrix $H_{U}$ associated to $\hat{\varphi}_{U}$ at the stationary point $\left(\bar{v}, \bar{x}_{0}, \bar{x}_{1}\right)$ takes the form

$$
H_{U}=\left(\begin{array}{ccc}
2 b p^{2} \ln ^{-1}(p / z) & 0 & -2 b p \ln ^{-1}(p / z) \\
0 & 1 / \bar{x}_{0}-2 & 0 \\
-2 b p \ln ^{-1}(p / z) & 0 & 2 b\left[\ln ^{-1}(p / z)-1\right]
\end{array}\right),
$$

one infers that $\bar{v}, \bar{x}_{0}$, and $\bar{x}_{1}$ lead to a minimum of replica energy when

$$
\bar{x}_{0}<1 / 2, \quad b<0, \quad p<z .
$$

The last two inequalities guarantee, from Eqs. (37) and (38), that $\bar{x}_{1}>0$ and $\bar{v}>0$; the latter inequality assures that the average volume actually contains $V_{0}$ in any possible equilibrium configuration. Moreover, Eq. (36) has two positive solutions if $0<z<z_{0}$ with $z_{0}=1 /(2 e) \approx 0.1839$, while no real solution exists for $z>z_{0}$. Notice that the smallest of the roots of Eq. (36) is that corresponding to $0<\bar{x}_{0}<1 / 2$. This implies that the fugacity cannot be arbitrarily large and that

$$
0<p<z<z_{0} .
$$

Therefore, we conclude that equilibrium configurations in the unconstrained ensemble can indeed be realized. In addition, we stress that there are no different states minimizing the replica energy for the same control parameters in the equilibrium region, hence the model has no phase transitions in the unconstrained ensemble. Furthermore, it is clear that Eqs. (37) and (38) are not well defined when $b=0$. As a consequence, the Thirring model $(b=0)$ does not attain equilibrium states in this ensemble; in this case, $T, P$, and $\mu$ cannot be taken as independent control parameters, just as it happens for macroscopic systems with short-range interactions. We will come back to this point in Sec. IV. Interestingly, we note that the condition $b<0$ means that the interactions within the outer 

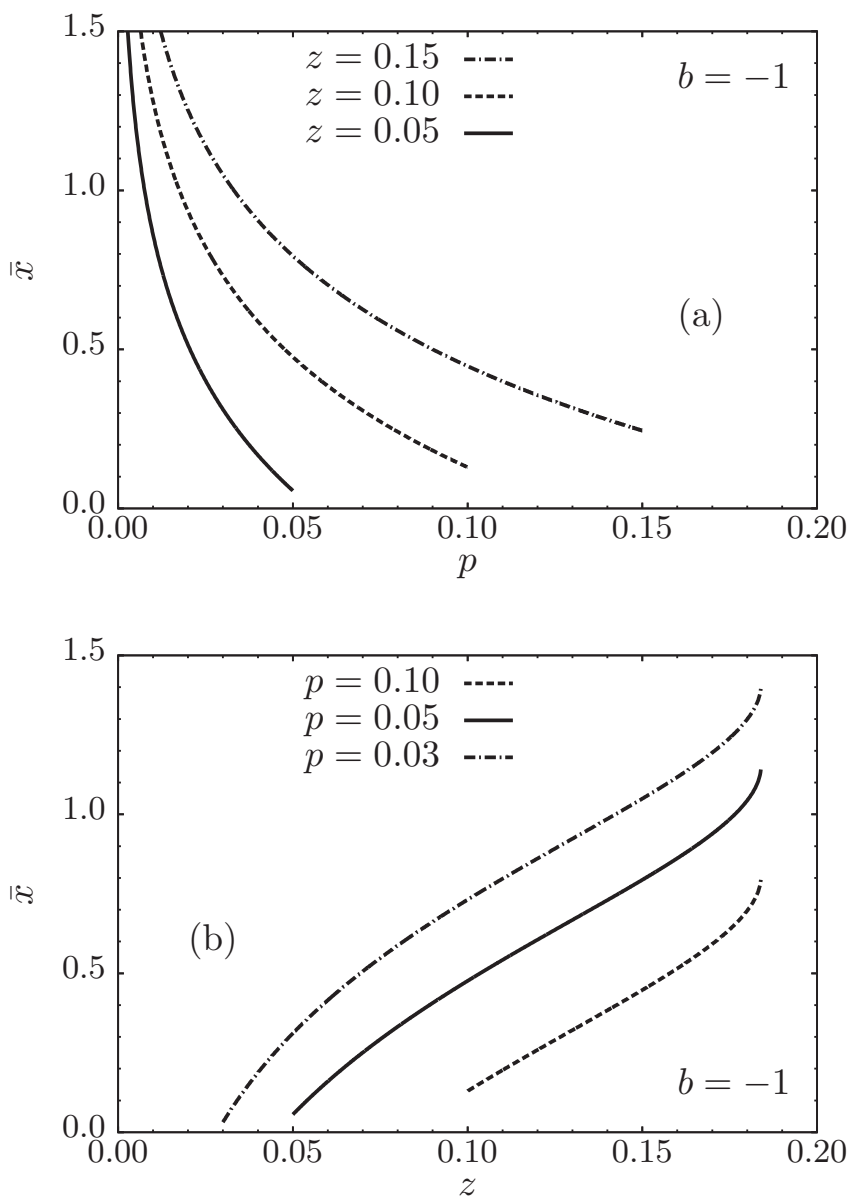

FIG. 1. Reduced number of particles $\bar{x}$ for the modified Thirring model with $b=-1$ in the unconstrained ensemble: in (a), as a function of the reduced pressure $p$ with constant fugacity $z$, and, in (b), as a function of the fugacity with constant reduced pressure. We note that in this and in the following four figures we have, as requested by the equilibrium condition in the unconstrained ensemble, $p<z$ and $z<1 /(2 e)$.

parts of the system must by repulsive to guarantee equilibrium configurations. This prevents the system from collapsing under completely open conditions in the appropriate range of control parameters.

In order to get further insight into the nature of the system, let us introduce the reduced number of particles $\bar{x}$ and reduced density $\bar{y}$ defined as

$$
\begin{gathered}
\bar{x}(p, z)=\frac{v \bar{N}}{T}=\bar{x}_{0}+\bar{x}_{1}, \\
\bar{y}(p, z)=\frac{v V_{0}}{T} \frac{\bar{N}}{\bar{V}}=\frac{\bar{x}_{0}+\bar{x}_{1}}{\bar{v}+1} .
\end{gathered}
$$

The behavior of these thermodynamic functions in the unconstrained ensemble can be quantitatively described for the model we are studying. Thus, the reduced number of particles $\bar{x}(p, z)$ is shown in Fig. 1(a) as a function of the reduced pressure $p$ with constant fugacity $z$, while in Fig. 1(b) it is plotted as a function of the fugacity with constant reduced pressure. In Fig. 2(a), the reduced density $\bar{y}(p, z)$ is represented as a function of the reduced pressure $p$ with constant fugacity
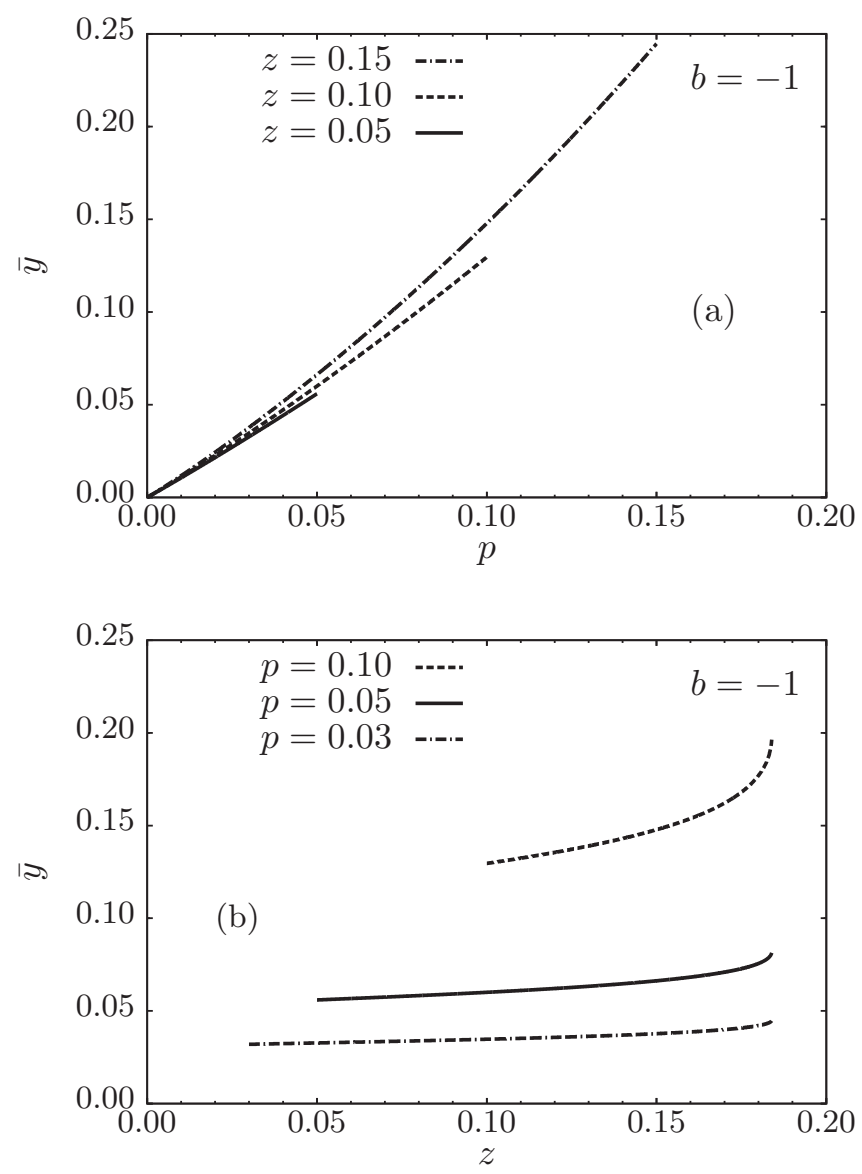

FIG. 2. Reduced density $\bar{y}$ for the modified Thirring model with $b=-1$ in the unconstrained ensemble: in (a), as a function of the reduced pressure $p$ with constant fugacity $z$, and, in (b), as a function of the fugacity with constant reduced pressure.

$z$, and, in Fig. 2(b), as a function of the fugacity with constant reduced pressure. Furthermore, in Fig. 3(a), we plot the reduced volume $\bar{v}(p, z)$, as given by Eq. (38), as a function of the reduced pressure $p$ for fixed values of the fugacity $z$. In Fig. 3(b), we show $\bar{v}$ as a function of $z$ holding the pressure constant. In all these plots, we have set the parameter $b$ of the model to $b=-1$.

Let us also consider the dimensionless response functions $q_{V}$ and $q_{N}$ defined according to

$$
\begin{aligned}
\frac{1}{q_{V}} & \equiv-\frac{T^{2}}{v V_{0}^{2}}\left(\frac{\partial \bar{V}}{\partial P}\right)_{T, \bar{N}}=-\left(\frac{\partial \bar{v}}{\partial p}\right)_{\bar{x}}, \\
\frac{1}{q_{N}} & \equiv v\left(\frac{\partial \bar{N}}{\partial \mu}\right)_{T, \bar{V}}=z(\bar{v}+1)\left(\frac{\partial \bar{y}}{\partial z}\right)_{\bar{v}}
\end{aligned}
$$

These response functions will be useful to compare the unconstrained ensemble with the canonical ensemble (see below), and we note that $q_{V}$ is related to the isothermal compressibility $\kappa_{T}$, according to

$$
\frac{1}{q_{V}}=\frac{V T^{2}}{v V_{0}^{2}} \kappa_{T} .
$$

In fact, here we are only interested in the signs of $q_{V}$ and $q_{N}$. Since $V$ and $P$, and $N$ and $\mu$, are conjugate variables, 

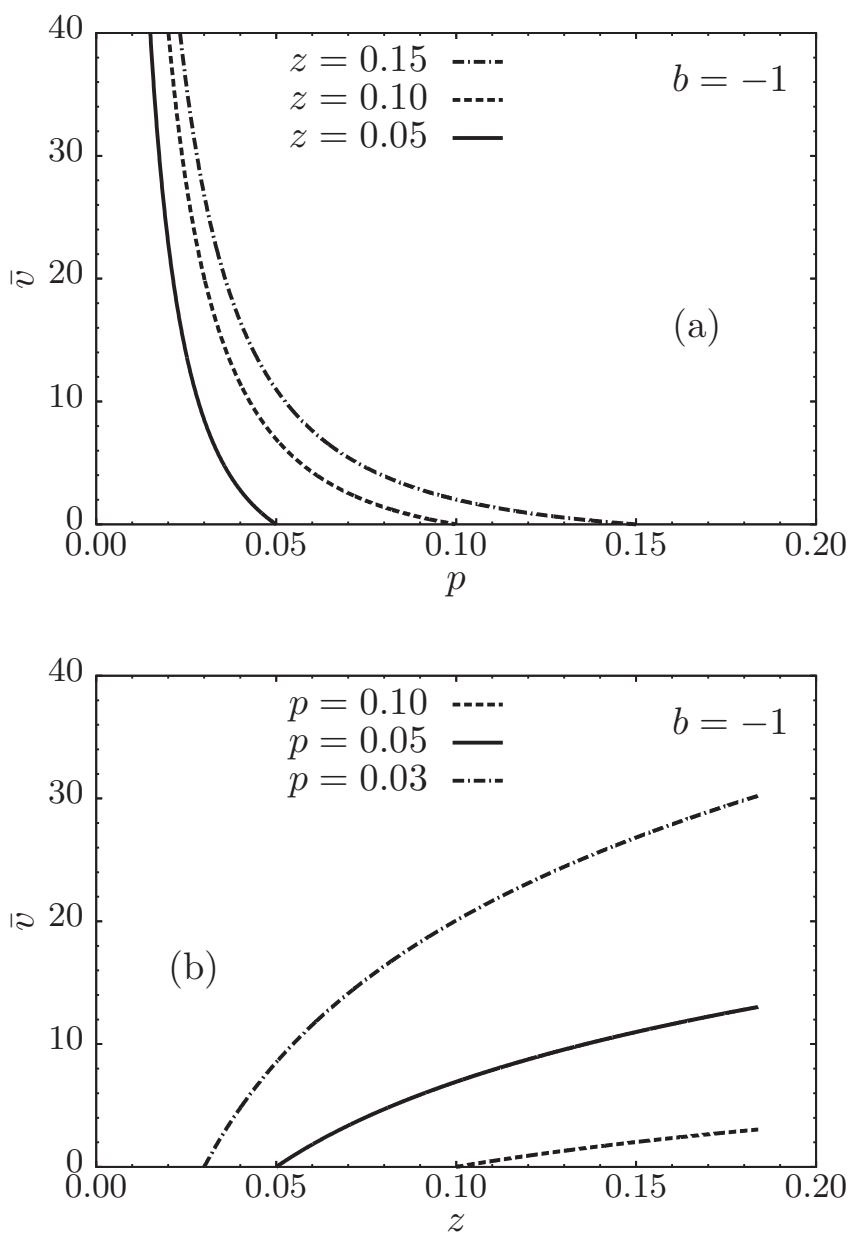

FIG. 3. Reduced volume $\bar{v}$ for the modified Thirring model with $b=-1$ in the unconstrained ensemble: in (a), as a function of the reduced pressure $p$ with constant fugacity $z$, and, in (b), as a function of the fugacity with constant reduced pressure.

respectively, and both the number of particles and the volume fluctuate here, the response functions $q_{V}$ and $q_{N}$ must be positive in this ensemble. Moreover, the signs of $q_{V}$ and $q_{N}$ can be seen by plotting the curves $\bar{v}$ vs. $p$ with constant $\bar{x}$, and $\bar{y}$ vs. $z$ with constant $\bar{v}$, respectively. Since $\bar{x}$ is not a control parameter, the curve $\bar{v}$ vs. $p$ with constant $\bar{x}$ represents the evolution of $\bar{v}$ as a function of $p$ through a series of equilibrium states where the actual control parameters, $p$ and $z$ in this case, are chosen in such a way that $\bar{x}$ takes the same value in all these states. This can be achieved if the reduced pressure is parametrized as

$$
p_{\bar{x}}(z)=z \exp \left\{2 b\left[\bar{x}-\bar{x}_{0}(z)\right]\right\}
$$

for the given $\bar{x}$, where we have used Eqs. (37) and (44). Moreover, since the reduced pressure is always lower than $z$ in equilibrium configurations, Eq. (49) must be restricted only to values of $z$ satisfying the condition $b\left[\bar{x}-\bar{x}_{0}(z)\right]<0$. Hence, the curve $\bar{v}$ vs. $p$ with constant $\bar{x}$ is obtained by parametrically plotting $p_{\bar{x}}(z)$ and $\bar{v}\left(p_{\bar{x}}(z), z\right)$ using $z$ as a parameter. The curve $\bar{y}$ vs. $z$ with constant $\bar{v}$ can be obtained in an analogous manner by choosing $p$ and $z$ in such a way that $\bar{v}$ takes always the same value. In this case, the reduced pressure is given by $p=p_{\bar{v}}(z)$,
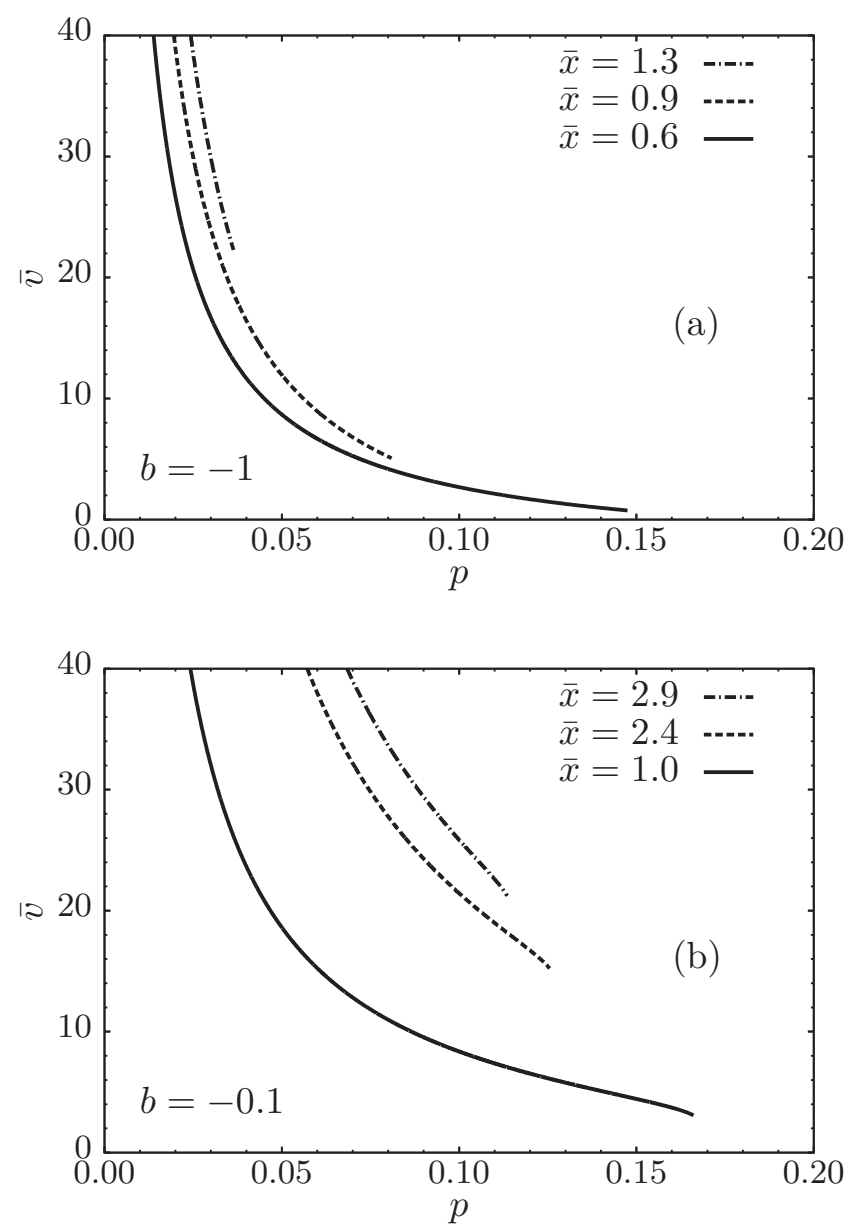

FIG. 4. (a) Reduced volume $\bar{v}$ as a function of the reduced pressure $p$ in the unconstrained ensemble, where $\bar{x}$ is held constant and $b=-1$.(b) Reduced volume as a function of the reduced pressure at constant $\bar{x}$ but with $b=-0.1$.

where, from Eq. (38), $p_{\bar{v}}(z)$ is the (numerical) solution of the equation

$$
\bar{v}=\frac{1}{2 b p_{\bar{v}}} \ln \left(\frac{p_{\bar{v}}}{z}\right)
$$

with fixed values of the reduced volume $\bar{v}$. As the reduced density $\bar{y}$ is given by Eq. (45) as a function of $p$ and $z$, the curve $\bar{y}$ vs. $z$ with constant $\bar{v}$ is obtained from Eq. (45) using $p=p_{\bar{v}}(z)$.

According to the previous discussion, in Fig. 4(a) and 4(b) we observe that $\bar{v}$ is a decreasing function of $p$ when $\bar{x}$ is fixed. This means that $q_{V}$ is positive for these configurations, as expected. Furthermore, as can be seen in Fig. 5(a) and 5(b), $\bar{y}$ increases for increasing $z$ with fixed $\bar{v}$, which indicates that the response function $q_{N}$ is also positive. Below we will show that the response functions in this model can be negative in the canonical ensemble, where $V$ and $N$ are fixed control parameters.

\section{B. The grand-canonical ensemble}

We now constrain the system by fixing the volume, so the control parameters in this case are $T, V$, and $\mu$, which 

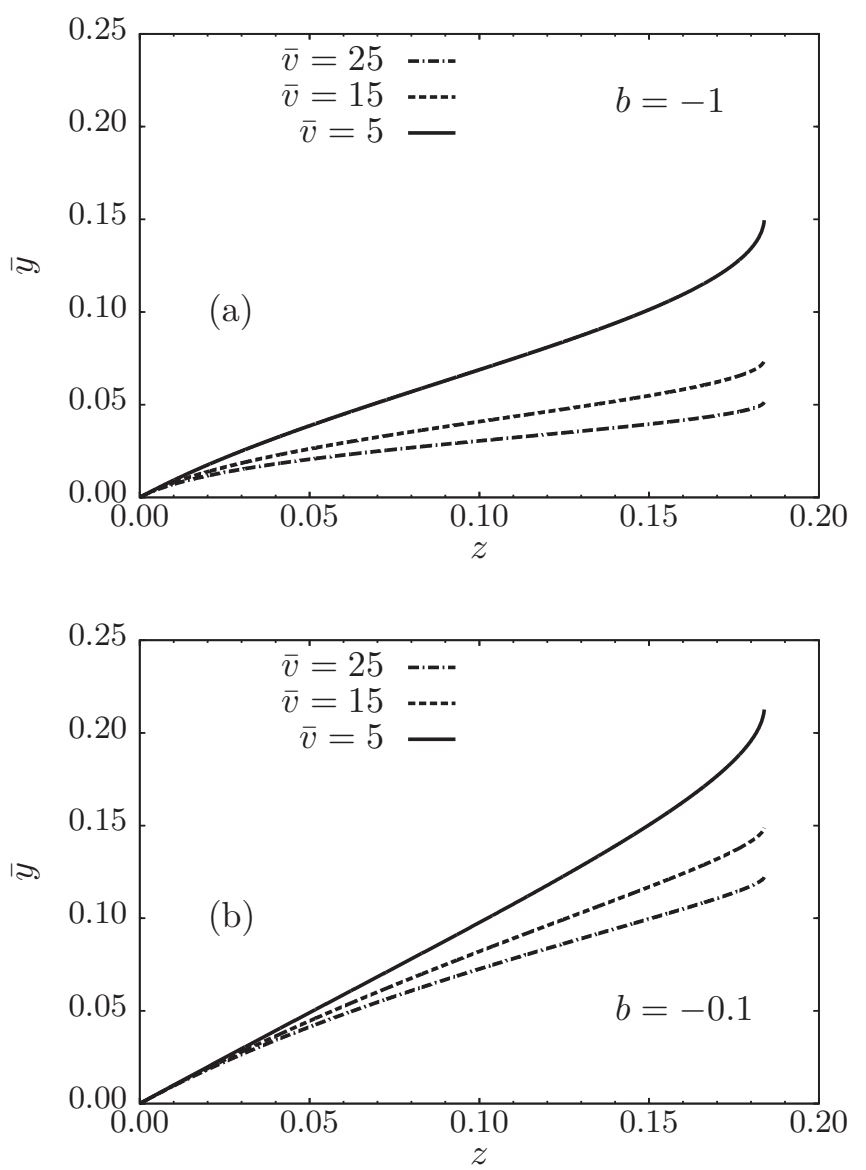

FIG. 5. (a) Reduced density $\bar{y}$ as a function of the fugacity $z$ in the unconstrained ensemble, where the reduced volume $\bar{v}$ is held constant and $b=-1$. (b) Reduced density as a function of the fugacity at constant $\bar{v}$ but with $b=-0.1$.

corresponds to the grand-canonical ensemble. Using Eq. (21), the grand-canonical partition function $\Xi=\sum_{N} e^{\beta \mu N} Z$ can be written as

$$
\Xi=\sum_{N} \int \frac{d^{3 N} \boldsymbol{q}}{N !} \lambda_{T}^{-3 N} e^{-\beta \hat{W}\left(N_{0}, N_{1}\right)} e^{\beta \mu N} .
$$

Thus, as before, replacing the integrals over positions by a sum over all possible values of the number of particles in the two regions, according to (23), one gets

$$
\Xi=\sum_{N_{0}, N_{1}} e^{-\beta \hat{\Omega}\left(N_{0}, N_{1}\right)},
$$

where, using Striling's approximation in the large- $N$ limit,

$$
\begin{aligned}
\hat{\Omega}\left(N_{0}, N_{1}\right)= & \hat{W}\left(N_{0}, N_{1}\right)-T \sum_{k} N_{k} \\
& +T \sum_{k} N_{k}\left[\ln \left(N_{k} \frac{\lambda_{T}^{3}}{V_{k}}\right)-\frac{\mu}{T}\right] .
\end{aligned}
$$

Using the saddle-point approximation, the grand potential is given by $\Omega=\inf _{\left\{N_{0}, N_{1}\right\}} \hat{\Omega}\left(N_{0}, N_{1}\right)$. The minimization with respect to $N_{0}$ and $N_{1}$ leads to

$$
\begin{gathered}
\mu=-2 \nu \bar{N}_{0}+T \ln \left(\frac{\bar{N}_{0}}{V_{0}} \lambda_{T}^{3}\right), \\
\mu=-2 b \nu \bar{N}_{1}+T \ln \left(\frac{\bar{N}_{1}}{V-V_{0}} \lambda_{T}^{3}\right),
\end{gathered}
$$

where now $\bar{N}_{0}$ and $\bar{N}_{1}$, being functions of $T, V$, and $\mu$, are the number of particles in each region that minimize the grand potential. The total mean number of particles is then given by $\bar{N}=\bar{N}_{0}+\bar{N}_{1}$. In addition, in the grand-canonical ensemble, the pressure is given by

$$
P=-\left(\frac{\partial \Omega}{\partial V}\right)_{T, \mu}=-\left(\frac{\partial \hat{\Omega}}{\partial V}\right)_{T, \mu},
$$

where the expression containing $\hat{\Omega}$ must be evaluated at $N_{0}=$ $\bar{N}_{0}$ and $N_{1}=\bar{N}_{1}$. Thus, for the modified Thirring model, one obtains

$$
P=\frac{T \bar{N}_{1}}{V-V_{0}}
$$

In addition, the replica energy in the grand-canonical ensemble is given by [30]

$$
\mathscr{E}=\Omega+P V=\Omega-V\left(\frac{\partial \Omega}{\partial V}\right)_{T, \mu} .
$$

This means that $\mathscr{E} \neq 0$ if $\Omega(T, V, \mu)$ is not a linear homogeneous function of $V$, and, therefore $\mathscr{E}$ vanishes only when the system is additive. From Eq. (58), using Eq. (53) evaluated at $N_{0}=\bar{N}_{0}$ and $N_{1}=\bar{N}_{1}$, one obtains

$$
\mathscr{E}=-W+P^{(e)} V
$$

with the excess pressure $P^{(e)}=P-\bar{N} T / V$. We stress that, in this case, the replica energy is a function of $T, V$, and $\mu$.

In order to study the equilibrium states of the system, we introduce the reduced grand potential $\varphi_{G}=\nu \Omega / T^{2}$ and the associated function $\hat{\varphi}_{G}=\nu \hat{\Omega} / T^{2}$, which are related through

$$
\varphi_{G}\left(\bar{x}_{0}, \bar{x}_{1}\right)=\inf _{\left\{x_{0}, x_{1}\right\}} \hat{\varphi}_{G}\left(x_{0}, x_{1}\right) .
$$

Here $x_{0}=v N_{0} / T$ and $x_{1}=v N_{1} / T$, so $\bar{x}_{0}=v \bar{N}_{0} / T$ and $\bar{x}_{1}=v \bar{N}_{1} / T$ are the corresponding quantities that minimize $\hat{\varphi}_{G}$, defining thus the equilibrium configurations in the grandcanonical ensemble. From Eq. (53), we obtain

$$
\begin{aligned}
\hat{\varphi}_{G}\left(x_{0}, x_{1}\right)= & x_{0}\left[\ln \left(\frac{x_{0}}{z}\right)-1\right]-x_{0}^{2} \\
& +x_{1}\left[\ln \left(\frac{x_{1}}{z v}\right)-1\right]-b x_{1}^{2},
\end{aligned}
$$

with the relative fugacity $z=e^{\left(\mu-\mu_{0}\right) / T}$ and the reduced volume $v=\left(V-V_{0}\right) / V_{0}$. Here the variables $z$ and $v$ can be taken as control parameters together with $T$. We note that, analogously to what occurred for the reduced replica energy in Sec. III A, the temperature $T$ does not appear explicitly in the reduced grand potential. In dimensionless variables, Eqs. (54) 
and (55) can be rewritten as

$$
\begin{gathered}
\bar{x}_{0}=z e^{2 \bar{x}_{0}}, \\
\bar{x}_{1}=z v e^{2 b \bar{x}_{1}} .
\end{gathered}
$$

Furthermore, the Hessian matrix $H_{G}$ associated to $\hat{\varphi}_{G}$ at the stationary point $\left(\bar{x}_{0}, \bar{x}_{1}\right)$ takes the form

$$
H_{G}=\left(\begin{array}{cc}
1 / \bar{x}_{0}-2 & 0 \\
0 & 1 / \bar{x}_{1}-2 b
\end{array}\right),
$$

and, therefore, one finds that $\hat{\varphi}_{G}$ can be minimized if

$$
\bar{x}_{0}<1 / 2, \quad 1 / \bar{x}_{1}>2 b .
$$

We thus observe that, as before, Eq. (62) has two solutions if $0<z<z_{0}$ with $z_{0}=1 /(2 e)$ and that the smallest of the roots of Eq. (62) is that corresponding to $0<\bar{x}_{0}<1 / 2$. Besides that, on the one hand, Eq. (63) has always one solution if $b \leqslant 0$. On the other hand, when $b>0$, Eq. (63) has two solutions if $0<z<z_{1}(v)$, where

$$
z_{1}(v)=\frac{1}{2 e b v},
$$

in such a way that the smallest of these solutions satisfies the condition (65). At $z=z_{1}(v)$, the only solution is given by $\bar{x}_{1}=1 /(2 b)$ and, hence, it corresponds to an unstable state. Therefore, Eqs. (62) and (63) can be solved simultaneously to give equilibrium configurations if

$$
0<z<z_{G}(v)
$$

where

$$
z_{G}(v)=\left\{\begin{array}{ll}
z_{0} & \text { if } b \leqslant 0 \\
\min \left[z_{0}, z_{1}(v)\right] & \text { if } b>0
\end{array} .\right.
$$

We note that for given values of the control parameters, the saddle-point equations define only one state, and, hence, there are no phase transitions in the grand-canonical ensemble. We also remark that the Thirring model $(b=0)$ attains equilibrium states in the grand-canonical ensemble if $0<z<z_{0}$.

In the grand-canonical ensemble, we now introduce the reduced number of particles, density, and pressure given by

$$
\begin{gathered}
\bar{x}(v, z)=\frac{v \bar{N}}{T}=\bar{x}_{0}+\bar{x}_{1}, \\
\bar{y}(v, z)=\frac{v V_{0}}{T} \frac{\bar{N}}{V}=\frac{\bar{x}_{0}+\bar{x}_{1}}{v+1}, \\
p(v, z)=\frac{v V_{0} P}{T^{2}}=\frac{\bar{x}_{1}}{v},
\end{gathered}
$$

respectively. In addition, the response functions are given by

$$
\begin{gathered}
q_{V}=-\frac{v V_{0}^{2}}{T^{2}}\left(\frac{\partial P}{\partial V}\right)_{T, \bar{N}}=-\left(\frac{\partial p}{\partial v}\right)_{\bar{x}} \\
\frac{1}{q_{N}}=v\left(\frac{\partial \bar{N}}{\partial \mu}\right)_{T, V}=z(v+1)\left(\frac{\partial \bar{y}}{\partial z}\right)_{v} .
\end{gathered}
$$

Hence, in view of Eq. (72), to put in evidence the sign of $q_{V}$, one has to plot $p$ as function of $v$ by holding $\bar{x}$ constant. Since $\bar{x}$ is not a control parameter in the grand-canonical ensemble, the curve $p$ vs. $v$ with constant $\bar{x}$ represents the evolution of $p$ as a function of $v$ through a series of equilibrium states characterized by the same $\bar{x}$. By combining Eqs. (62) and (63), $z$ can in fact be chosen in such a way that $\bar{x}$ remains constant when $v$ is varied. Using these values of $z$ in Eq. (71), we obtain the curve $p$ vs. $v$ with constant $\bar{x}$. Moreover, the sign of $q_{N}$ can be directly seen by plotting $z$ vs. $\bar{y}$ at constant $v$, since $\bar{y}=\bar{y}(v, z)$ as given by Eq. (70).

For $b<0$, the pressure-volume relation is invertible in the grand-canonical ensemble. That is, from Eqs. (63) and (71), we can write

$$
v=\frac{1}{2 b p(v, z)} \ln \left[\frac{p(v, z)}{z}\right],
$$

which for constant $z$ defines the same relation as in the unconstrained ensemble. Moreover, the equilibrium conditions are the same in the two ensembles for $b<0$. Therefore, in the modified Thirring model, the grand-canonical and unconstrained ensembles are equivalent when $b<0$.

Since also the class of models with $b \geqslant 0$ attains equilibria in the grand-canonical ensemble, the phenomenology in this case is richer than in the unconstrained case. For instance, equilibrium configurations with $p \geqslant z$ or with negative isothermal compressibility cannot be observed under completely open conditions, while these configurations can be realized with fixed volume. In Fig. 6(a), we show $p$ as a function of $z$ with fixed $v$ for different values of the parameter $b$. It can be seen that $p>z$ when $b>0$, while $p<z$ when $b<0$ as it happens in the unconstrained ensemble. In addition, in this plot we observe that $p=z$ for $b=0$, a general feature of the Thirring model. All the curves in Fig. 6(a) finish at $z=z_{G}(v)$, since beyond this critical fugacity the stability is lost in the grand-canonical ensemble. In Fig. 6(b), $p$ is plotted as a function of $v$ by holding $\bar{x}$ constant, where also different values of $b$ are chosen. Since holding $\bar{x}$ constant determines the value of the fugacity when $v$ is varied, the curves start at the minimum value of $v$ for which the condition $0<z<z_{G}(v)$ is satisfied, ensuring thus the equilibrium of the configurations. As a remarkable fact, a region where $q_{V}<0$ is observed for $b=0.5$, which corresponds to the points of the curve with positive slope. Configurations in such a region have negative isothermal compressibility.

\section{The canonical ensemble}

In order to understand better the behavior of the system in the unconstrained ensemble, it is instructive to compare its equilibrium states with the corresponding ones in the canonical ensemble. Hence, we consider now that the control parameters are $T, V$, and $N$. Using (23) and the integral representation

$$
\delta_{N, N_{0}+N_{1}}=\int_{\alpha-i \pi}^{\alpha+i \pi} \frac{d \zeta}{2 \pi i} e^{\zeta\left(N-N_{0}-N_{1}\right)}
$$

with $\operatorname{Re}[\zeta]=\alpha=-\mu / T$, the canonical partition function (21) can be written as

$$
Z=\int_{\alpha-i \pi}^{\alpha+i \pi} \frac{d \zeta}{2 \pi i} \sum_{N_{0}, N_{1}} e^{-\hat{F}\left(\zeta, N_{0}, N_{1}\right) / T}
$$



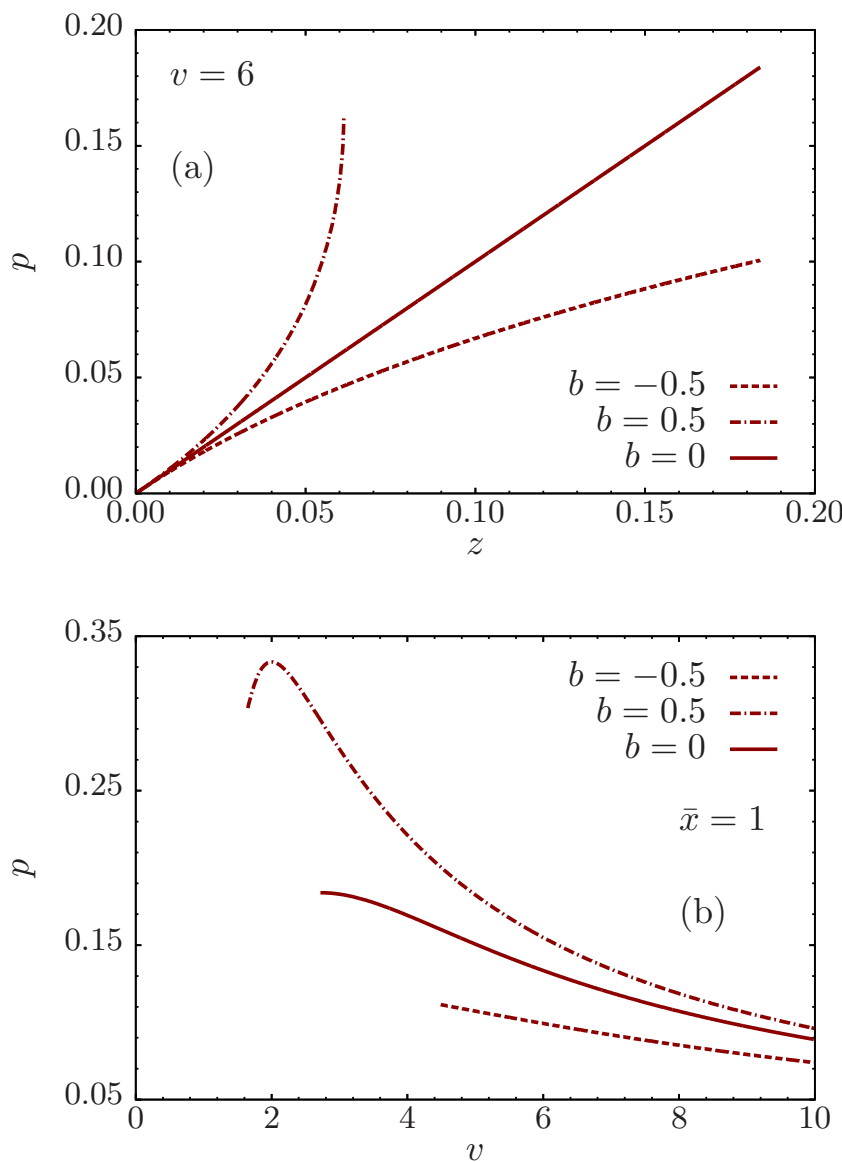

FIG. 6. Modified Thirring model in the grand-canonical ensemble. (a) Reduced pressure as a function of the fugacity for constant reduced volume $v$. For $b<0$, the reduced pressure is always smaller than the fugacity, while, for $b>0$, it is shown that $p>z$. In the case $b=0$, the condition $p=z$ is always satisfied. (b) Pressure as a function of the volume with constant reduced number of particles $\bar{x}$. In the case $b=0.5$, the portion of the curve with positive slope correspond to states of negative isothermal compressibility.

where, for large $N$,

$$
\begin{aligned}
\hat{F}\left(\zeta, N_{0}, N_{1}\right)= & -\zeta T\left(N-\sum_{k} N_{k}\right)+\hat{W}\left(N_{0}, N_{1}\right) \\
& +T \sum_{k} N_{k}\left[\ln \left(\frac{N_{k}}{V_{k}} \lambda_{T}^{3}\right)-1\right] .
\end{aligned}
$$

The canonical Helmholtz free energy is thus given by $F=$ $\inf _{\left\{\alpha, N_{0}, N_{1}\right\}} \hat{F}\left(\alpha, N_{0}, N_{1}\right)$, where $\zeta$ is evaluated at its real part $\alpha$. Moreover, minimization with respect to $\alpha$ enforces that $\bar{N}_{1}=N-\bar{N}_{0}$, while minimization with respect to $N_{0}$ and $N_{1}$ leads to

$$
\begin{gathered}
\mu=-2 v \bar{N}_{0}+T \ln \left(\frac{\bar{N}_{0}}{V_{0}} \lambda_{T}^{3}\right), \\
\mu=-2 v b\left(N-\bar{N}_{0}\right)+T \ln \left(\frac{N-\bar{N}_{0}}{V-V_{0}} \lambda_{T}^{3}\right),
\end{gathered}
$$

where we have used that $\alpha=-\mu / T$, and where the bars denote that the corresponding quantity minimizes the canonical free energy. In view of

$$
\mu=\left(\frac{\partial F}{\partial N}\right)_{T, V}=\left(\frac{\partial \hat{F}}{\partial N}\right)_{T, V},
$$

with the expression containing $\hat{F}$ evaluated at $N_{0}=\bar{N}_{0}$ and $N_{1}=\bar{N}_{1}$, we note that $\mu$ is indeed the chemical potential of the system that, in the canonical ensemble, is no more a control parameter. Equating the right-hand sides of Eqs. (78) and (79), we get an equation for $\bar{N}_{0}$ as a function of $T, V$, and $N$. Furthermore, the pressure is given by

$$
P=-\left(\frac{\partial F}{\partial V}\right)_{T, N}=-\left(\frac{\partial \hat{F}}{\partial V}\right)_{T, N},
$$

so

$$
P=\frac{T \bar{N}_{1}}{V-V_{0}} .
$$

We now turn our attention to the replica energy in the canonical ensemble. It is given by [30]

$$
\begin{aligned}
\mathscr{E} & =F+P V-\mu N \\
& =F-V\left(\frac{\partial F}{\partial V}\right)_{T, N}-N\left(\frac{\partial F}{\partial N}\right)_{T, V},
\end{aligned}
$$

so now $\mathscr{E} \neq 0$ if $F(T, V, N)$ is not a linear homogeneous function of $V$ and $N$, and it vanishes only when the system is additive. From Eqs. (83) and (77), we again obtain

$$
\mathscr{E}=-W+P^{(e)} V
$$

where $P^{(e)}=P-N T / V$.

Furthermore, taking $x=v N / T$ and $v=\left(V-V_{0}\right) / V_{0}$ as control parameters, and, as before, introducing $\bar{x}_{0}=v \bar{N}_{0} / T$ in the canonical ensemble, Eqs. (78) and (79) can be combined into

$$
2 b x-2(1+b) \bar{x}_{0}+\ln \left(\frac{\bar{x}_{0}}{x-\bar{x}_{0}}\right)+\ln v=0 .
$$

Notice that $\bar{x}_{0} / x=\bar{N}_{0} / N$ represents the fraction of particles inside the volume $V_{0}$, so $0 \leqslant \bar{x}_{0} / x \leqslant 1$. Equation (85) defines $\bar{x}_{0}=\bar{x}_{0}(x, v)$ in this ensemble, and, depending on the parameters, it can have two solutions. Again, in reduced variables, the temperature does not appear. The solution determining the equilibrium states corresponds to $x_{0}=\bar{x}_{0}$ that minimizes the canonical free energy or, equivalently, the reduced free energy $\varphi_{C}=v F / T^{2}$. From Eq. (77) and according to the variational problem for this case, one obtains

$$
\varphi_{C}\left(\bar{x}_{0}\right)=\inf _{x_{0}} \hat{\varphi}_{C}\left(x_{0}\right)
$$

with

$$
\begin{aligned}
\hat{\varphi}_{C}\left(x_{0}\right)= & 2 b x x_{0}-(1+b) x_{0}^{2}+x_{0} \ln \left(\frac{x_{0}}{x-x_{0}}\right) \\
& +x \ln \left(1-\frac{x_{0}}{x}\right)+x_{0} \ln v,
\end{aligned}
$$

where in Eq. (87) we have used that $\bar{N}_{1}=N-\bar{N}_{0}$ and omitted terms that do not depend on $x_{0}$. It is interesting to observe that Eq. (85) can have more than one solution, so the model may exhibit phase transitions. In the Appendix, we discuss how to 
obtain the critical point for the modified Thirring model in the canonical ensemble.

Once (85) is solved satisfying (86), the relative fugacity $z=$ $e^{\left(\mu-\mu_{0}\right) / T}$ can be computed from Eq. (78), since this equation can be rewritten as

$$
z(x, v)=\bar{x}_{0} e^{-2 \bar{x}_{0}}
$$

Furthermore, the reduced pressure $p=v V_{0} P / T^{2}$ takes the form

$$
p(x, v)=\frac{x-\bar{x}_{0}}{v} .
$$

The response functions, as defined in Sec. III A, in the canonical ensemble are given by

$$
\begin{gathered}
q_{V}=\frac{v V_{0}^{2}}{T^{2}}\left(\frac{\partial^{2} F}{\partial V^{2}}\right)_{T, N}=-\left(\frac{\partial p}{\partial v}\right)_{x}, \\
q_{N}=\frac{1}{v}\left(\frac{\partial^{2} F}{\partial N^{2}}\right)_{T, V}=\frac{1}{z(v+1)}\left(\frac{\partial z}{\partial y}\right)_{v},
\end{gathered}
$$

where we have introduced the reduced density

$$
y=\frac{v V_{0}}{T} \frac{N}{V}=\frac{x}{v+1} .
$$

As discussed in Sec. III A, the sign of the response functions $q_{V}$ and $q_{N}$ can be be inferred from the slopes of the curves $p(v)$ at constant $x$ and $z(y)$ at constant $v$, respectively. Here we want to compare these curves with the corresponding ones in the unconstrained ensemble. We do this for negative values of $b$, for which the unconstrained and the grand-canonical ensembles are equivalent. Then the curves represent also the comparison between the canonical and grand-canonical ensembles when $b<0$. Thus, in Fig. 7(a), we show $p$ as a function of $\bar{v}$ in the unconstrained ensemble with $b=-1$, where $\bar{x}$ is held constant. The corresponding curves in the canonical ensemble are also shown in this plot, where $v$ and $x$ are fixed to the same values of $\bar{v}$ and $\bar{x}$, respectively. In Fig. 7(b), these curves are represented for $b=-0.1$ and different values of $\bar{x}$, where it can be appreciated that the model presents first-order phase transitions in the canonical ensemble, as indicated by the jumps in the pressure. In addition, in Fig. 8(a) we represent $z$ as a function of $\bar{y}$ in the unconstrained ensemble, where $\bar{v}$ is held constant and we set $b=-1$. We also show the analogous curves in the canonical ensemble, where $y$ and $v$ are fixed to the same values of $\bar{y}$ and $\bar{v}$, respectively. In Fig. 8(b), these curves are shown for $b=-0.1$. The jumps in the fugacity correspond to first-order phase transitions in the canonical ensemble. Thus, the canonical and unconstrained ensembles are nonequivalent at the macrostate level $[38,39]$, since the equilibrium states in the two ensembles are not in one-to-one correspondence. In particular, we highlight that the response functions can be negative in the canonical ensemble. Finally, we briefly note that a nonequivalence between the microcanonical and canonical ensembles is expected for the modified Thirring model, since this happens for the particular case $b=0$ [40].
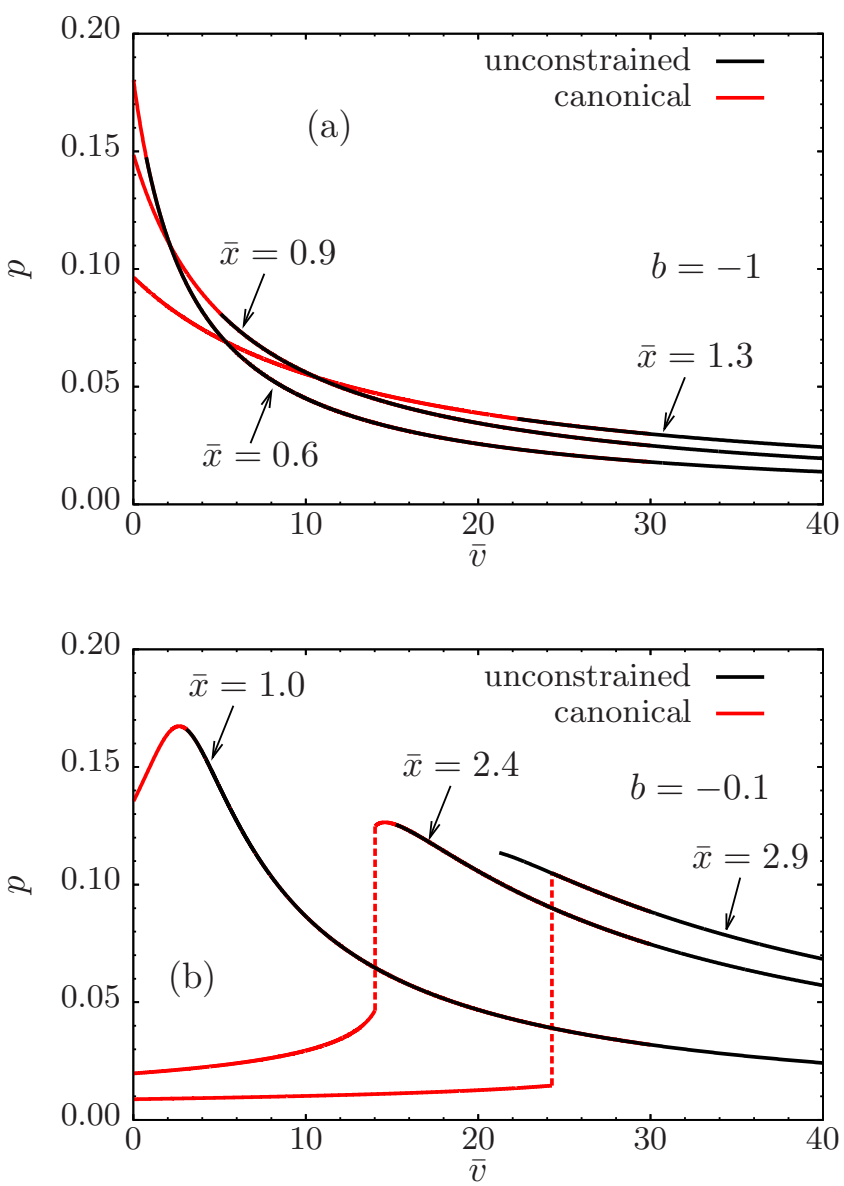

FIG. 7. Comparison between the unconstrained and the canonical ensembles in the modified Thirring model. (a) The black lines indicate the reduced pressure $p$ as a function of the reduced volume $\bar{v}$ in the unconstrained ensemble, where the reduced number of particles $\bar{x}$ is held constant for $b=-1$. The red lines show the analogous curves in the canonical ensemble, where $v$ and $x$ are fixed to the same values of $\bar{v}$ and $\bar{x}$, respectively. (b) The plot shows the same as in (a) but with $b=-0.1$ and different values of $\bar{x}$. For $b=-0.1$, the model shows a first-order phase transition in the canonical ensemble, as can be appreciated in the plot from the jumps in pressure (red dotted lines). In both (a) and (b), the curves in the canonical ensemble continue to the right superposed on the curves in the unconstrained ensemble. We remark that in (b), the three curves in the canonical ensemble present a portion with positive slope, indicating negative compressibility. In particular, the slopes of the curves with $\bar{x}=2.4$ and $\bar{x}=2.9$ are positive before the jump.

\section{THERMODYNAMIC RELATIONS AND REPLICA ENERGY}

In Sec. II, from an equation for the differential variations of the replica energy in the unconstrained ensemble, we have obtained a set of relations in terms of partial derivatives that allows one to obtain, e.g., the entropy of the system. Let us recall these relations since they will be the central issue of the following discussion:

$$
d \mathscr{E}=-S d T+V d P-N d \mu
$$



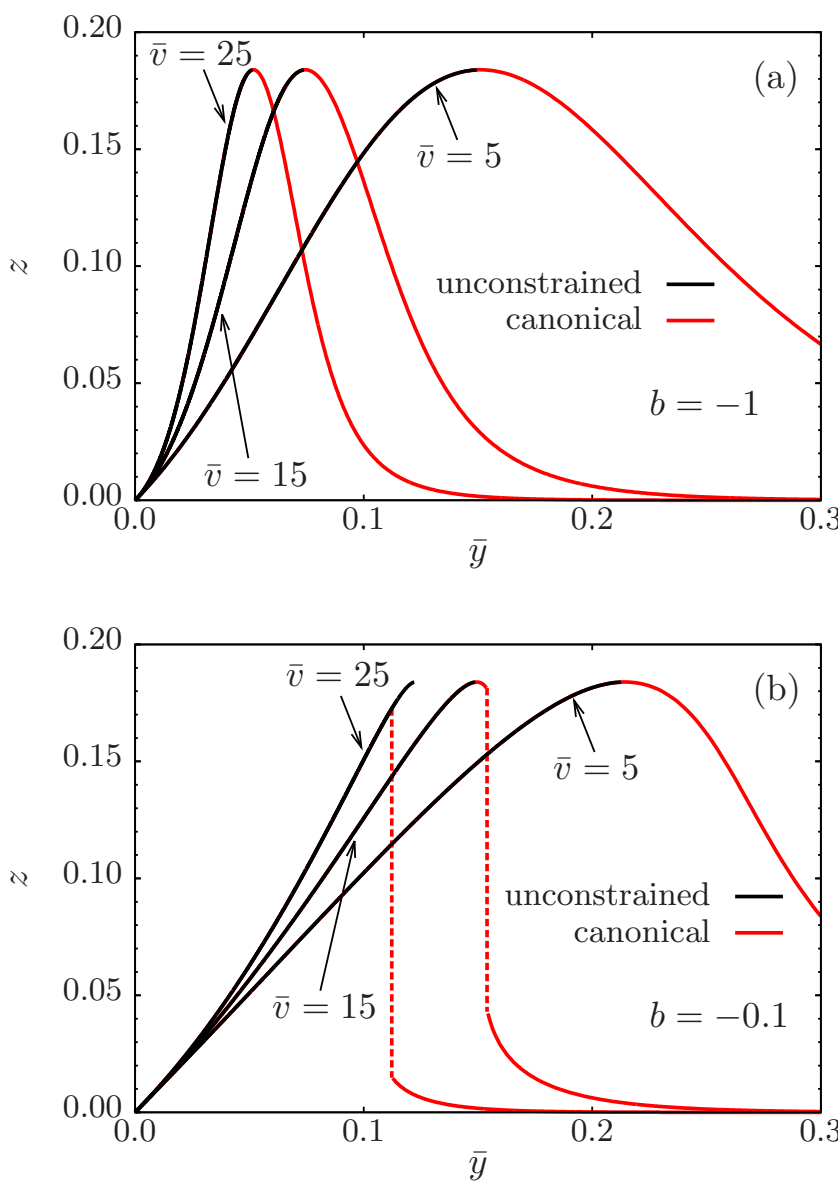

FIG. 8. Comparison between the unconstrained and the canonical ensembles in the modified Thirring model. (a) The black lines represent the fugacity $z$ as a function of the reduced density $\bar{y}$ in the unconstrained ensemble. Here the reduced volume $\bar{v}$ is held constant and we set $b=-1$. The red lines show the analogous curves in the canonical ensemble, where $y$ and $v$ are fixed to the same values of $\bar{y}$ and $\bar{v}$, respectively. In (b), the plot shows the same as in (a) but with $b=-0.1$. The jumps correspond to first-order phase transitions in the canonical ensemble (red dotted lines). In both (a) and (b), the curves in the canonical ensemble continue to the left superposed on the curves in the unconstrained ensemble.

and

$$
\begin{gathered}
\left(\frac{\partial \mathscr{E}}{\partial T}\right)_{P, \mu}=-S, \\
\left(\frac{\partial \mathscr{E}}{\partial P}\right)_{T, \mu}=V, \\
\left(\frac{\partial \mathscr{E}}{\partial \mu}\right)_{T, P}=-N .
\end{gathered}
$$

These equations, however, are thermodynamic relations valid in any ensemble; with this in mind, we do not use here a bar over the variables to indicate whether a certain quantity fluctuates.

The present discussion is to emphasize that a situation may exist in which the replica energy differs from zero, but $T, P$, and $\mu$ cannot be taken as a set of independent variables. This can happen in an ensemble that differs from the unconstrained one. When $T, P$, and $\mu$ cannot be taken as independent variables in the unconstrained ensemble, the mean-field equations will not lead to a minimum of replica energy, and, therefore, Eqs. (93)-(96) are meaningless.

Let us assume that the system is itself constrained such that, for instance, $\mu=\mu(T, P)$. In this case, Eq. (93) becomes

$$
d \mathscr{E}=-\left[S+N\left(\frac{\partial \mu}{\partial T}\right)_{P}\right] d T+\left[V-N\left(\frac{\partial \mu}{\partial P}\right)_{T}\right] d P
$$

which establishes a functional relation $\mathscr{E}=\mathscr{E}(T, P)$. Thus, one actually has

$$
\begin{gathered}
\left(\frac{\partial \mathscr{E}}{\partial T}\right)_{P}=-S-N\left(\frac{\partial \mu}{\partial T}\right)_{P}, \\
\left(\frac{\partial \mathscr{E}}{\partial P}\right)_{T}=V-N\left(\frac{\partial \mu}{\partial P}\right)_{T},
\end{gathered}
$$

which are the relations satisfied in this case instead of Eqs. (94) and (95). This is equivalent to directly consider a constraint $\mathscr{E}=\mathscr{E}(T, P)$ in Eq. (93), which yields

$$
N d \mu=-\left[S+\left(\frac{\partial \mathscr{E}}{\partial T}\right)_{P}\right] d T+\left[V-\left(\frac{\partial \mathscr{E}}{\partial P}\right)_{T}\right] d P \text {. }
$$

The above expression shows that the usual Gibbs-Duhem equation is not valid when the replica energy differs from zero even if $T, P$, and $\mu$ are not a set of independent variables. Moreover, analogous equations can be obtained if one considers any other functional relation constraining $T, P$, and $\mu$.

To go further, consider an arbitrary $d$-dimensional system with long-range interactions whose number density at a point $\boldsymbol{x} \in \mathbb{R}^{d}$ is given by

$$
n(\boldsymbol{x})=\frac{1}{\lambda_{T}^{d}} \exp \left[\frac{\mu-\Phi(\boldsymbol{x})}{T}\right],
$$

where $\Phi(\boldsymbol{x})$ is the mean-field potential. The chemical potential and the entropy of the system satisfy [25]

$$
\begin{aligned}
\mu N & =T \int n(\boldsymbol{x}) \ln \left[n(\boldsymbol{x}) \lambda_{T}^{d}\right] d^{d} \boldsymbol{x}+2 W, \\
-S & =\int n(\boldsymbol{x}) \ln \left[n(\boldsymbol{x}) \lambda_{T}^{d}\right] d^{d} \boldsymbol{x}-\frac{2+d}{2} N,
\end{aligned}
$$

where $W=\frac{1}{2} \int n(\boldsymbol{x}) \Phi(\boldsymbol{x}) d^{d} \boldsymbol{x}$ is the potential energy. Moreover, the local pressure is given by $p(x)=n(x) T$ when shortrange interactions are completely ignored, and the pressure $P$ is $p(x)$ evaluated at the boundary of the system [24]. Consider also that the mean-field potential is not completely arbitrary, but it always vanishes at the boundary of the system, regardless of the thermodynamic state of the system. This will enforce the condition

$$
\mu=T \ln \left(\frac{P \lambda_{T}^{d}}{T}\right)
$$

Thus, using Eq. (104) in Eqs. (98) and (99), and then using Eqs. (102) and (103), leads to

$$
\left(\frac{\partial \mathscr{E}}{\partial T}\right)_{P}=-\frac{2 W}{T}
$$




$$
\left(\frac{\partial \mathscr{E}}{\partial P}\right)_{T}=\frac{P^{(e)} V}{P},
$$

where $P^{(e)}=P-N T / V$. Hence, with the particular constraint (104), Eqs. (105) and (106) hold in place of Eqs. (94) and (95).

The interesting fact here is that the modified Thirring model can be used to test these general considerations. In Sec. III A we found that in the case $b=0$ the model never attains equilibrium configurations in the unconstrained ensemble. As we shall see below, this is due to the fact that, for the case $b=0$, the chemical potential satisfies Eq. (104) (with $d=3$ ), and thus $T, P$, and $\mu$ cannot be taken as independent variables. But first we will check that for $b \neq 0$ the entropy can be computed from Eq. (94), showing that $T, P$, and $\mu$ can actually be taken as independent variables in this case. This, of course, is in agreement with the statistical mechanics description of the system obtained in Sec. III A.

To check the validity of Eq. (94), we need to write the replica energy as a function of $T, P$, and $\mu$. We note that $\Phi(\boldsymbol{x})=-2 v\left[N_{0} \theta_{V_{0}}(\boldsymbol{x})+b N_{1} \theta_{V_{1}}(\boldsymbol{x})\right]$ and $N_{1} / V_{1}=P / T$ for the modified Thirring model. Thus, evaluating the number density (101) at any point $\boldsymbol{x}$ in $V_{1}$ and rearranging terms gives us

$$
\mu=T \ln \left(\frac{P \lambda_{T}^{3}}{T}\right)-2 \nu b N_{1},
$$

which defines $N_{1}=N_{1}(T, P, \mu)$ if $b \neq 0$. Analogously, evaluating (101) at any point $\boldsymbol{x}$ in $V_{0}$ yields

$$
\mu=T \ln \left(\frac{N_{0} \lambda_{T}^{3}}{V_{0}}\right)-2 \nu N_{0},
$$

which defines implicitly $N_{0}=N_{0}(T, \mu)$. Therefore, when $b \neq$ 0 , we have

$$
\begin{aligned}
& \left(\frac{\partial N_{0}}{\partial T}\right)_{P, \mu}=\frac{2 v N_{0}^{2}+\mu N_{0}-\frac{3}{2} N_{0} T}{\left(2 v N_{0}-T\right) T}, \\
& \left(\frac{\partial N_{1}}{\partial T}\right)_{P, \mu}=\frac{N_{1}}{T}+\frac{1}{2 b v}\left(\frac{\mu}{T}-\frac{5}{2}\right) .
\end{aligned}
$$

Since

$$
\begin{aligned}
\mathscr{E} & =-W+P^{(e)} V \\
& =v\left(N_{0}^{2}+b N_{1}^{2}\right)+P V_{0}-N_{0} T,
\end{aligned}
$$

the replica energy is implicitly given as a function of $T, P$, and $\mu$ by means of Eqs. (107) and (108). Hence,

$$
\left(\frac{\partial \mathscr{E}}{\partial T}\right)_{P, \mu}=\sum_{k} N_{k}\left[\ln \left(\frac{N_{k} \lambda_{T}^{3}}{V_{k}}\right)-\frac{5}{2}\right],
$$

where we have rearranged terms using Eqs. (107) and (108). By comparing with Eq. (103), we thus see that the right-hand side of Eq. (112) is indeed $-S$. This confirms that when $b \neq 0$, the system has enough thermodynamic degrees of freedom to take $T, P$, and $\mu$ as a set of independent variables. To see whether the configurations are stable or not, however, one must perform an analysis of the second-order variations of the appropriate free energy, as we have done in Sec. III A, for instance, depending on the actual physical conditions imposed by the corresponding control parameters.
In view of Eq. (107), it is now obvious that if $b=0$, the chemical potential is given by Eq. (104) and that now

$$
2 v N_{0}=T \ln \left(\frac{N_{0} T}{P V_{0}}\right)
$$

defines implicitly $N_{0}=N_{0}(T, P)$. Hence,

$$
\left(\frac{\partial N_{0}}{\partial T}\right)_{P}=\frac{N_{0}}{T}\left(\frac{2 v N_{0}+T}{2 v N_{0}-T}\right)
$$

Using Eq. (114) and since in this case $\mathscr{E}=v N_{0}^{2}+P V_{0}-$ $N_{0} T$, it is easy to see that

$$
\left(\frac{\partial \mathscr{E}}{\partial T}\right)_{P}=\frac{2 v N_{0}^{2}}{T}=-\frac{2 W}{T}
$$

in agreement with Eq. (105). These arguments show that, although the replica energy differs from zero, in the Thirring model $(b=0)$ one cannot take $T, P$, and $\mu$ as a set of independent variables.

\section{v. CONCLUSIONS}

We have shown that systems with long-range interactions can attain configurations of thermodynamic equilibrium in the unconstrained ensemble. In this ensemble, the control parameters are temperature, pressure, and chemical potential and the appropriate free energy is the replica energy. We have presented a solvable model that is stable in this ensemble, and we have compared its equilibrium states with those of the grand-canonical and canonical ensembles. From this comparison, we observe that the space of parameters defining the possible stable configurations is enlarged when the system is constrained by fixing the volume and the number of particles. These quantities fluctuate in the unconstrained ensemble, as well as the energy of the system. Moreover, the model we have introduced exhibits first-order phase transitions in the canonical ensemble at which the system undergoes pressure and fugacity jumps.

On the one hand, macroscopic systems with short-range interactions cannot attain equilibrium states if the control parameters are the temperature, pressure, and chemical potential. According to the usual Gibbs-Duhem equation, these variables cannot be taken as independent. A physical reason behind this property is that, in this case, temperature, pressure, and chemical potential are truly intensive properties and, therefore, they cannot define the size of the system corresponding to an equilibrium state. On the other hand, however, in systems with long-range interactions, temperature, pressure, and chemical potential are not intensive properties, so controlling, e.g., the temperature $T \sim N$, can actually define the size of the system in an equilibrium configuration. Thus, the typical scaling of the thermodynamic variables in long-range interacting systems makes it possible to have equilibrium configurations in completely open conditions. 


\section{ACKNOWLEDGMENTS}

I.L. acknowledges financial support through an FPI scholarship (BES-2012-054782) from the Spanish Government under Project No. FIS2011-22603.

\section{APPENDIX: CRITICAL POINT OF THE MODIFIED THIRRING MODEL IN THE CANONICAL ENSEMBLE}

Phase transitions in the modified Thirring model in the canonical ensemble can be studied by extending the analysis done in Ref. [40] using Landau theory of phase transitions. In Ref. [40], the expansion parameter specifying the transition is taken as the deviation of the fraction $1-N_{0} / N$ with respect to the value of this fraction at the transition line. Here, equivalently, we consider the fraction of particles in $V_{0}$, given by $x_{0} / x$, and take $m=\left(x_{0}-\bar{x}_{0}\right) / x$ as the expansion parameter. Accordingly, to obtain the critical point, we expand the free energy (87) as $\hat{\varphi}_{C}=\varphi_{0}+\varphi_{1} m+$ $\varphi_{2} m^{2}+\mathcal{O}\left(m^{3}\right)$ and look for a solution of the system of equations

$$
\varphi_{1}\left(\bar{x}_{0}, x, v\right)=0, \quad \varphi_{2}\left(\bar{x}_{0}, x, v\right)=0,
$$

with the constraint $0 \leqslant \bar{x}_{0} / x \leqslant 1$. The first of Eqs. (A1) is exactly (85), while the second one can be written as

$$
\frac{2(b+1)\left(x-\bar{x}_{0}\right) \bar{x}_{0}-x}{\left(x-\bar{x}_{0}\right) \bar{x}_{0}}=0 .
$$

Thus, for $b>-1$, Eq. (A2) has two real solutions, $\bar{x}_{0}=x_{ \pm}(x)=[1+b \pm \sqrt{(1+b)(1+b-2 / x)}]^{-1}$, when $x>$ $2 /(1+b) \equiv x_{c}$. The critical point is defined by the condition [40] $x_{+}\left(x_{c}\right)=x_{-}\left(x_{c}\right)$, in such a way that at the critical point one has $x=x_{c}$ and $\bar{x}_{0}=x_{ \pm}\left(x_{c}\right)=x_{c} / 2$. In terms of the thermodynamic variables, this means that the critical temperature is given by $T_{c}=v N(1+b) / 2$ and that the fraction $\bar{N}_{0} / N$ at the critical point is $1 / 2$. Moreover, we note that when $b=-1$, the left-hand side of Eq. (A2) does not vanish, and, therefore, there are no phase transitions in this case. For $b<-1$, the fractions $x_{ \pm} / x$ do not lie in the interval $[0,1]$, so also in this case the model will not exhibit phase transitions. We therefore observe that the model may present phase transitions in the canonical ensemble, depending on the value of $v$, if $x$ is larger than $x_{c}$ and if $b>-1$.

The critical value of the reduced volume $v_{c}$ is obtained by replacing $\bar{x}_{0}=x_{ \pm}\left(x_{c}\right)$ in Eq. (85) with $v=v_{c}$, yielding $v_{c}=\exp [2(1-b) /(1+b)]$. Thus, the model may exhibit phase transitions when $v>v_{\mathrm{c}}$.
[1] A. Campa, T. Dauxois, D. Fanelli, and S. Ruffo, Physics of LongRange Interacting Systems (Oxford University Press, Oxford, 2014); A. Campa, T. Dauxois, and S. Ruffo, Phys. Rep. 480, 57 (2009).

[2] F. Bouchet, S. Gupta, and D. Mukamel, Physica A 389, 4389 (2010).

[3] Y. Levin, R. Pakter, F. B. Rizzato, T. N. Teles, and F. P. C. Benetti, Phys. Rep. 535, 1 (2014).

[4] V. A. Antonov, Vest. Leningrad Gros. Univ. 7, 135 (1962); Trans. IAU Symposium 113, 525 (1985).

[5] D. Lynden-Bell and R. Wood, Mon. Not. R. Astron. Soc. 138, 495 (1968).

[6] W. Thirring, Z. Phys. 235, 339 (1970).

[7] T. Padmanabhan, Phys. Rep. 188, 285 (1990).

[8] D. Lynden-Bell, Physica A 263, 293 (1999).

[9] P.-H. Chavanis, Astron. Astrophys. 381, 340 (2002).

[10] H. de Vega and N. Sánchez, Nucl. Phys. B 625, 409 (2002); 625, 460 (2002).

[11] P.-H. Chavanis, Int. J. Mod. Phys. B 20, 3113 (2006).

[12] M. K. H. Kiessling and T. Neukirch, Proc. Natl. Acad. Sci. USA 100, 1510 (2003).

[13] D. R. Nicholson, Introduction to Plasma Theory (Wiley, New York, 1983).

[14] P.-H. Chavanis and J. Sommeria, Phys. Rev. E 65, 026302 (2002).

[15] R. Robert and J. Sommeria, J. Fluid. Mech. 229, 291 (1991).

[16] T. Mori, J. Stat. Mech. (2013) P10003.

[17] Fernanda P. da C. Benetti, T. N. Teles, R. Pakter, and Y. Levin, Phys. Rev. Lett. 108, 140601 (2012).

[18] Y. Y. Yamaguchi, J. Barré, F. Bouchet, T. Dauxois, and S. Ruffo, Physica A 337, 36 (2004).
[19] R. S. Ellis, K. Haven, and B. Turkington, J. Stat. Phys. 101, 999 (2000).

[20] J. Barré, D. Mukamel, and S. Ruffo, Phys. Rev. Lett. 87, 030601 (2001).

[21] F. Bouchet and J. Barré, J. Stat. Phys. 118, 1073 (2005).

[22] P. Chomaz and F. Gulminelli, in Dynamics and Thermodynamics of Systems with Long-Range Interactions, Lecture Notes in Physics Vol. 602, edited by T. Dauxois, S. Ruffo, E. Arimondo, and M. Wilkens (Springer, New York, 2002), p. 68.

[23] M. Schmidt, R. Kusche, T. Hippler, J. Donges, W. Kronmüller, B. von Issendorff, and H. Haberland, Phys. Rev. Lett. 86, 1191 (2001).

[24] I. Latella and A. Pérez-Madrid, Phys. Rev. E 88, 042135 (2013).

[25] I. Latella, A. Pérez-Madrid, A. Campa, L. Casetti, and S. Ruffo, Phys. Rev. Lett. 114, 230601 (2015).

[26] L. Velazquez, J. Stat. Mech. (2016) 033105.

[27] Y. Levin, R. Pakter, and T. N. Teles, Phys. Rev. Lett. 100, 040604 (2008).

[28] T. L. Hill, Statistical Mechanics: Principles and Selected Applications (Dover, New York, 1987).

[29] E. A. Guggenheim, J. Chem. Phys. 7, 103 (1939); R. H. Fowler and E. A. Guggenheim, Statistical Thermodynamics (Cambridge University Press, London, 1939).

[30] T. L. Hill, Thermodynamics of Small Systems, Parts I and II (Dover, New York, 2013).

[31] T. L. Hill and R. V. Chamberlin, Proc. Natl. Acad. Sci. USA 95, 12779 (1998).

[32] T. L. Hill, Nano Lett. 1, 273 (2001).

[33] T. L. Hill and R. V. Chamberlin, Nano Lett. 2, 609 (2001).

[34] S. K. Schnell, T. J. Vlugt, J.-M. Simon, D. Bedeaux, and S. Kjelstrup, Chem. Phys. Lett. 504, 199 (2011). 
[35] S. K. Schnell, T. J. Vlugt, J.-M. Simon, D. Bedeaux, and S. Kjelstrup, Mol. Phys. 110, 1069 (2012).

[36] E. A. Guggenheim, Thermodynamics: An Advanced Treatment for Chemists and Physicists (North-Holland, Amsterdam, 1967).

[37] A. Planes and E. Vives, J. Stat. Phys. 106, 827 (2002).
[38] H. Touchette, R. S. Ellis, and B. Turkington, Physica A 340, 138 (2004).

[39] H. Touchette, J. Stat. Phys. 159, 987 (2015).

[40] A. Campa, L. Casetti, I. Latella, A. Pérez-Madrid, and S. Ruffo, J. Stat. Mech. (2016) 073205. 